\title{
INFLUENCE OF DIFFERENT AIR ABRASION PRESSURE ON SHEAR BOND STRENGTH OF DIFFERENT TYPES OF ZIRCONIA
}

\author{
Ehab A. Farghaly* and Rania Reda Afifi**
}

\begin{abstract}
Aim: This study aimed to evaluate the use of different pressure of air particle abrasion (APA) on four different types of monolithic zirconia and its effect on their shear bond strength.

Methodology: The study was divided into 4 groups according to the type of monolithic zirconia used: opaque, high translucency, ultra-high translucency and supra-high translucency. Each group was subdivided into two groups according to the amount of APA application used (2 or 4 bars). For the treatment of the zirconia specimens, zirconia MDP containing primer was applied on the cemented surface. Composite resin cubes were fabricated and cemented over zirconia cubes under $5 \mathrm{~kg}$ for 6 minutes. Shear bond strength test was carried out on an Instron machine at a speed of $0.5 \mathrm{~mm} / \mathrm{min}$, with the load measured in Newtons and the SBS determined as follows: Load $(\mathrm{N}) /$ area $=$ SBS. Effect of APA application were examined using scanning electron microscopy to determine surface roughness on different tested zirconia groups. The data was statistically analized.
\end{abstract}

Results: The one way ANOVA test revealed statistical differences between the APA pressure groups $(\mathrm{P}<0.001)$. Statistical analysis showed that high translucent zirconia scored the highest shear bond after using 2 bar and 4 bar APA in comparison to all other groups; the shear bond strength increased with increasing the pressure.

Conclusion: The lower values of shear bond strength in successive, more translucent generations, forewarning the necessity for greater caution in clinical implementation.

Keywords: Air particle abrasion ,monolithic zirconia , shear bon strength, scanning electron microscope

\section{INTRODUCTION}

Dentistry is both art and science updated practitioner need to be adaptable and ready to versatile. In terms of cosmetic restorations, the dental profession continues to make milestones. Practitioner commonly faces the dilemma of which material to be used when restoring a tooth. The aesthetics and strength of the prostheses, as well as providing a conservative planning, are major factors that can affect the final decision (Cardoso et al. 2012). Zirconia has recently gained popularity due to its

\footnotetext{
* Lecturer Prosthetic Dentistry Department, Faculty of Oral \&Dental Medicine, Nahda University, Beni Suef, Egypt.

** Lecturer Conservative Dentistry Department, Faculty of Dentistry, Alexandria University, Alexandria, Egypt.
} 
non-metallic appearance, durability, and remarkable fracture resistance (flexural strength of over 1,000 MPa).(Matsuzaki et al. 2015).

Zirconia contains over $90 \%$ zirconium oxide without silica, which provides more fracture toughness and strength compared to other ceramic materials. Unlike other silica-based ceramics, that had several drawbacks such as delamination that was solved by the introduction of the monolithic zirconia, which had the problem of opacity that was solved by the translucent zirconia, which in turn have a critical weakness forming durable and reliable bonds due to its resistance to acid etching especially when talking about zirconia veneers (Nistor et al. 2019).

Despite that the different surface treatments increased the bond strength to different types of zirconia available such as the opaque, high translucency ultra-high translucency and suprahigh translucency zirconia to the underlying tooth structure, still the bond strength is not comparable between these types and even to glass infiltrated ceramics (Ågren et al 2019). The problem of debonding of zirconia veneers regardless of the bonding technique has made zirconia less usable although it has the best strength of about 1000 MPa (Thompson et al. 2011). Alumina air-abrasion is recommended through recent investigations to enhance bond strength of resin cement to zirconia; as it increases the surface area for micro-mechanical interlocking (Yang et al. 2010; El Zayat et al. 2020; Moon et al. 2016).

In this study, the use of different pressure of air particle abrasion (APA) on different types of zirconia was performed, to test its subsequent effect on shear bond strength.

The null hypothesis was that the different APA pressure will not alter the shear bond strength of all types of tested zirconia.

\section{MATERIAL AND METHODS}

\section{Specimen grouping}

The study was divided into 4 groups ( $\mathrm{n}=20$ each): according to the type of zirconia used: opaque (KATANA zirconia ML), high translucency (KATANA zirconia HT), ultra- translucency (KATANA Zirconia UTML) and supra- translucency (Katana Zirconia STML). According to the amount of APA application used, each group was divided into two groups ( 2 or 4 bars).

\section{Specimen preparation}

\section{Zirconia ceramic specimens}

Specimens $4 \times 4$ of the material (3D cubes $6 \times 6 \mathrm{~mm}$, thickness of $3 \mathrm{~mm}$ ) were drawn using AUTOCAD (Autodesk, mac, 2017), and after being exported as STL (standard triangulation language) file to CAD/ CAM software (Dwos software, Weiland Dental) the 4 types of blocks were simultaneously mounted in the milling machine (Weiland Zenostar coping, Weiland Dental) where 20 cubes were milled from each zirconia block in dry mode. The cubes were cleaned with jets of air and then placed in ultrasonic solution for 20 secs and left to dry.

All samples were then placed in ceramic oven for sintering according to manufacturer directions: temperature of $1550^{\circ} \mathrm{C}$ with increasing rate of $10^{\circ} \mathrm{c} / \mathrm{min}$, holding time 2 hours then decreasing temperature rate is $10^{\circ} \mathrm{c} / \mathrm{min}$.

Each group was subdivided into two groups $(\mathrm{n}=10)$ to receive air particle abrasion by $\mathrm{Al} 2 \mathrm{O} 3$ $(50 \mu \mathrm{m})$ one group received 2 bars and the other group received 4 bars.

The micro-etcher of the sandblasting unit was fixed at angle of the 60 degree to the specimens and at a distance of $1 \mathrm{~cm}$ away from the tip of the micro etcher.

\section{Resin blocks specimens}

A cuboid of $4 \times 4$ and thickness of $3 \mathrm{~mm}$ was cut into a transparent plexi frame, and another plexi of the size of the original was used to act as a base for 
the cuboid. The two plexi's placed on top of each other and Ceram.x (Dentsply Sirona) composite was packed in the cuboid. It was insured that composite has a flat surface using a histology glass and then the composite was cured. The plexi's are then separated and standardized $4 \times 4$ composite cubes are obtained.

\section{Cementation}

For the treatment of the zirconia specimens, zirconia primer containing MDP monomer (Clearfil Ceramic Primer Plus) was applied on the cemented surface, left for 10 seconds and thinned out by air. Regarding the composite cubes, bonding agent (3M ESPE, USA) was applied to their cemented surface.

On the primed surface, Breeze's dual cure selfetch self-adhesive resin cement (Breeze, USA) was used. In the plexi frames, composite resin cubes were cemented in exact position over zirconia cubes under $5 \mathrm{~kg}$ for 10 minutes.

\section{Specimen testing}

A plexi framework with $6 \mathrm{~mm}$ thickness was fabricated to hold the specimen while testing with a cuboid cut in the center of $6 \times 6$ dimensions and cemented to a base of another plexi. The framework was placed longitudinally in the cuboid cut, thus the tip of the universal testing machine (Instron 8874, Instron Corp.) will fall on the interface of cube/ composite until sudden drop of load (Fig 1). The shear bond strength was measured by the Instron machine at a speed of $0.5 \mathrm{~mm} / \mathrm{min}$, the load recorded in Newtons and SBS calculated as follows SBS $(\mathrm{MPa})=\operatorname{load}(\mathrm{N}) / \operatorname{area}\left(\mathrm{mm}^{2}\right)$.

Data was analysed using IBM SPSS software package version 20.0. The Kolmogorov-Smirnov test was used to ensure that the distribution of variables was normal. For normally distributed quantitative variables, the student t-test was used to compare the groups. ANOVA was used to compare the various studied groups, followed by a pairwise analysis using the Post Hoc test (Tukey). The significance of the obtained findings was assessed at a $5 \%$ level of significance.

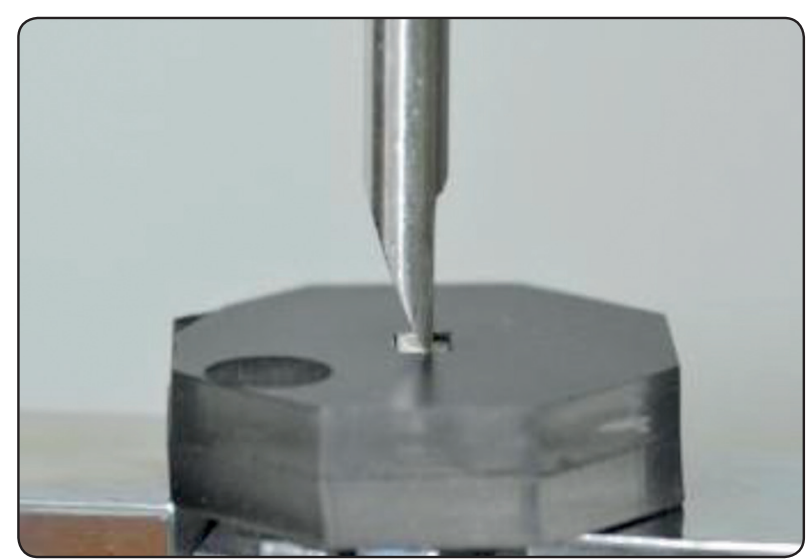

Fig. (1) Shear bond strength test

\section{Scanning electron microscope:}

Surface images of each zirconia group specimens were recorded using Quanta FEG 250 scanning electron microscope (FEI Company, USA).

\section{RESULTS}

\section{Shear bond strength}

Statistical analysis was conducted to evaluate the effect of air particle abrasion on the shear bond strength between different types of zirconia.

Table 1 shows the means and standard deviations of the shear bond strength values. The one way ANOVA test revealed statistical differences between the APA pressure groups $(\mathrm{P}<0.001)$.

Statistical analysis showed that high translucent zirconia scored the highest SBS values under 2 and 4 bars air particle abrasion. Statistically significant difference was found between opaque and high translucent zirconia under 2 and 4 bar pressure with $\mathrm{p}$ value 0.003 and 0.015 respectively as well as between high and ultra, high and supra translucent zirconia as well under 2 bar pressure with $\mathrm{p}$ values 0.012 and 0.001 respectively, and between high and ultra-translucent under 4 bar pressure as well with $\mathrm{p}$ value 0.03 . Opaque zirconia showed statistically significant different between the values of 2 and 4 bars pressure $(\mathrm{p}=0.028)$ while supra translucent zirconia showed the same $(\mathrm{p}=0.001)$ (Figure 2). 
TABLE (1) Comparison between the different types of zirconia according to shear bond strength

\begin{tabular}{|c|c|c|c|c|c|}
\hline Shear bond strength & $\begin{array}{c}\text { Opaque } \\
(\mathbf{O}) \\
(\mathbf{n}=\mathbf{1 0})\end{array}$ & $\begin{array}{c}\text { High Translucent } \\
(\mathbf{H}) \\
(\mathbf{n = 1 0})\end{array}$ & $\begin{array}{c}\text { Ultra Translucent } \\
(\mathbf{U}) \\
(\mathbf{n}=\mathbf{1 0})\end{array}$ & $\begin{array}{c}\text { Supra } \\
\text { Translucent }(\mathbf{S}) \\
(\mathbf{n}=\mathbf{1 0})\end{array}$ & $(\mathbf{p})$ \\
\hline $\mathbf{2}$ bars (Mean \pm SD) & $23.2^{\mathrm{b}} \pm 4.9$ & $33.2^{\mathrm{a}} \pm 8.4$ & $24.4^{\mathrm{b}} \pm 4.7$ & $21.8^{\mathrm{b}} \pm 5.4$ & $\left(0.001^{*}\right)$ \\
\hline $\mathbf{4}$ bars $($ Mean \pm SD) & $28.7^{\mathrm{b}} \pm 5.5$ & $37.6^{\mathrm{a}} \pm 8.6$ & $29.5^{\mathrm{b}} \pm 6.3$ & $30.7^{\mathrm{ab}} \pm 3.4$ & $\left(0.011^{*}\right)$ \\
\hline${ }^{\mathrm{t}}$ & $0.028^{*}$ & 0.265 & 0.053 & $0.001^{*}$ & \\
\hline
\end{tabular}

Means in the same raw with common small letters are not significant.

$p: p$ value for comparing between the different types of zirconia

${ }^{t} p$ : $p$ value for Student $t$-test for comparing between 2 bars and 4 bars in each type

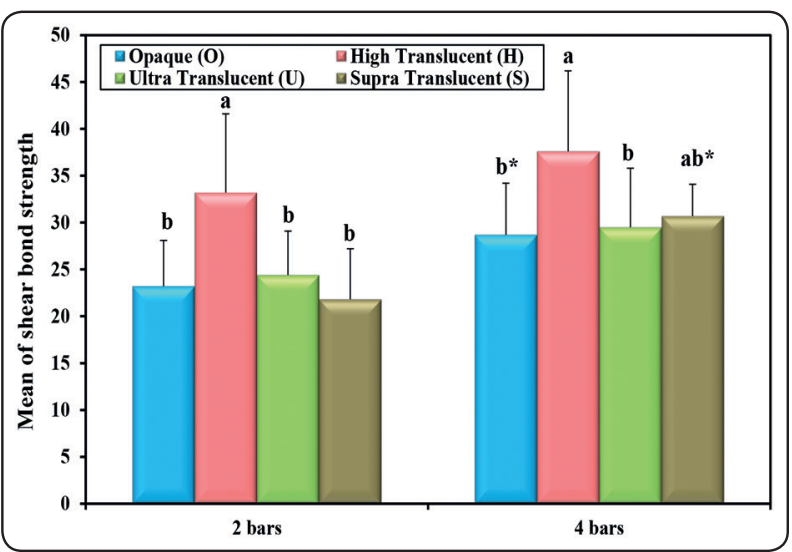

Fig. (2) Comparison between the different types of zirconia according to shear bond strength./Common small letters are not significant, different small letters are significant *: the 4 bar values are significant with the 2 bar ones

\section{Scanning electron microscope (SEM)}

SEM images $(\times 1000)$ of the treated zirconia surfaces among tested groups using 2 and 4 bars air particle abrasion with $\mathrm{Al}_{2} \mathrm{O}_{3}(50 \mu \mathrm{m})$ showed in Figs 3,4 . The 2 bars air particle abrasion surface treatment resulted in the creation of shallow striations in opaque zirconia. While, high translucent zirconia showed more roughness with many deep irregularities. Ultra translucent zirconia surface showed relatively roughness with shallow striations and pores and Supra translucent zirconia showed more smooth surface with less irregularities (Fig.3). Air born particle abrasion with 4 bars resulted in creation of multiple striated shallow grooves in Opaque zirconia. While, high translucent zirconia showed very rough surface with multiple deep grooves and deeper pores. Ultra translucent zirconia showed relatively roughness with micro-retentive grooves and Supra translucent zirconia showed many rough cracks with multiple relatively deep scars and grooves (Fig.4).

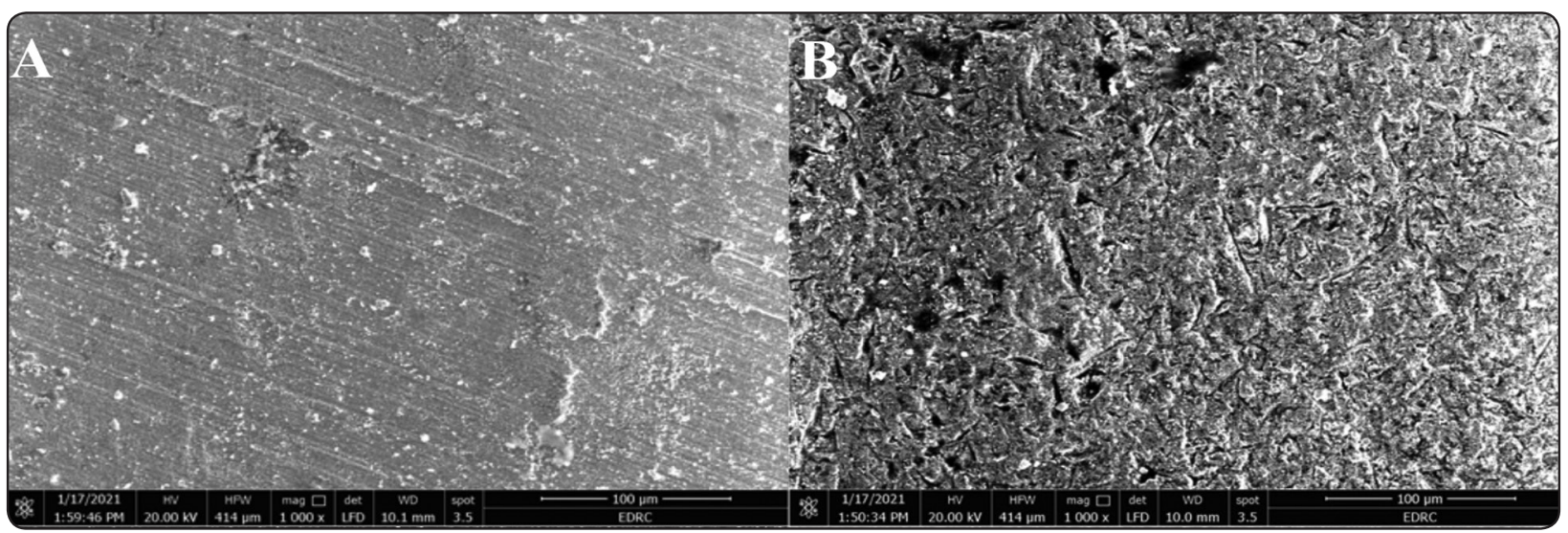




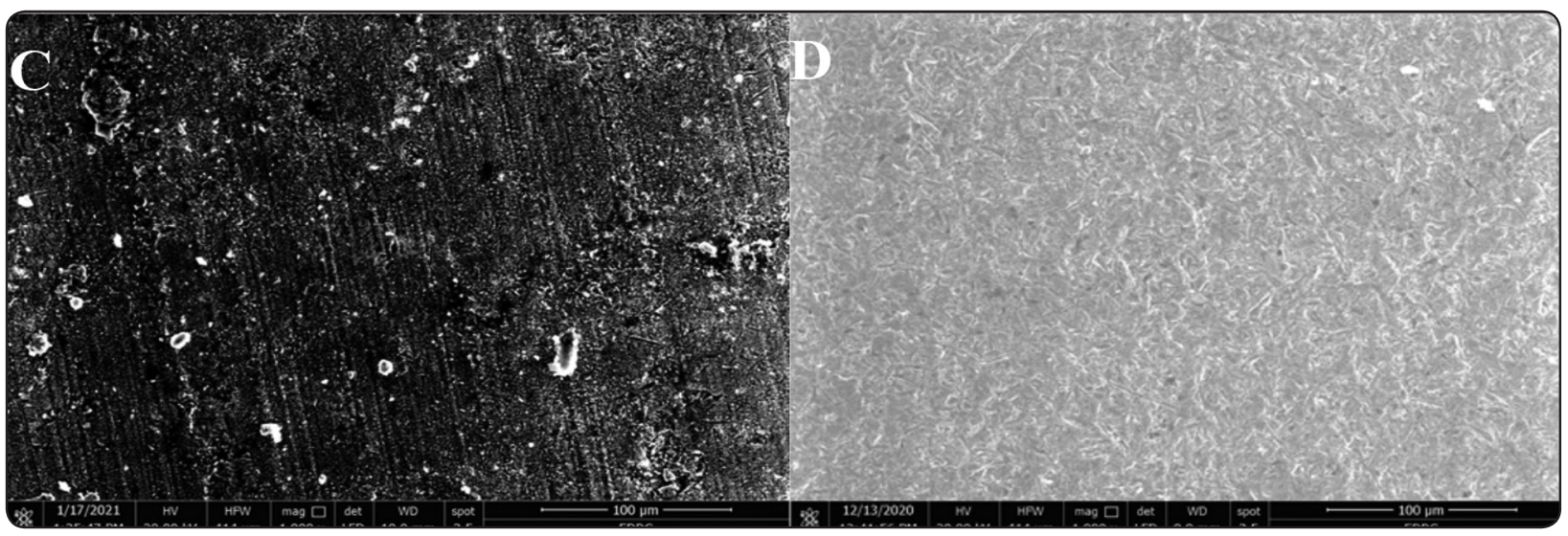

Fig. (3) Scanning electron microscope photos represents surface topography of zirconia specimens with 2 bars air particle abrasion. A: Opaque zirconia; the surface showed shallow striations. B: High translucent zirconia; the surface showed more roughness with many deep irregularities. C: Ultra translucent Zirconia; the surface showed relatively roughness with shallow striations and pores. D: Supra translucent zirconia; the surface showed more smooth surface with less irregularities.

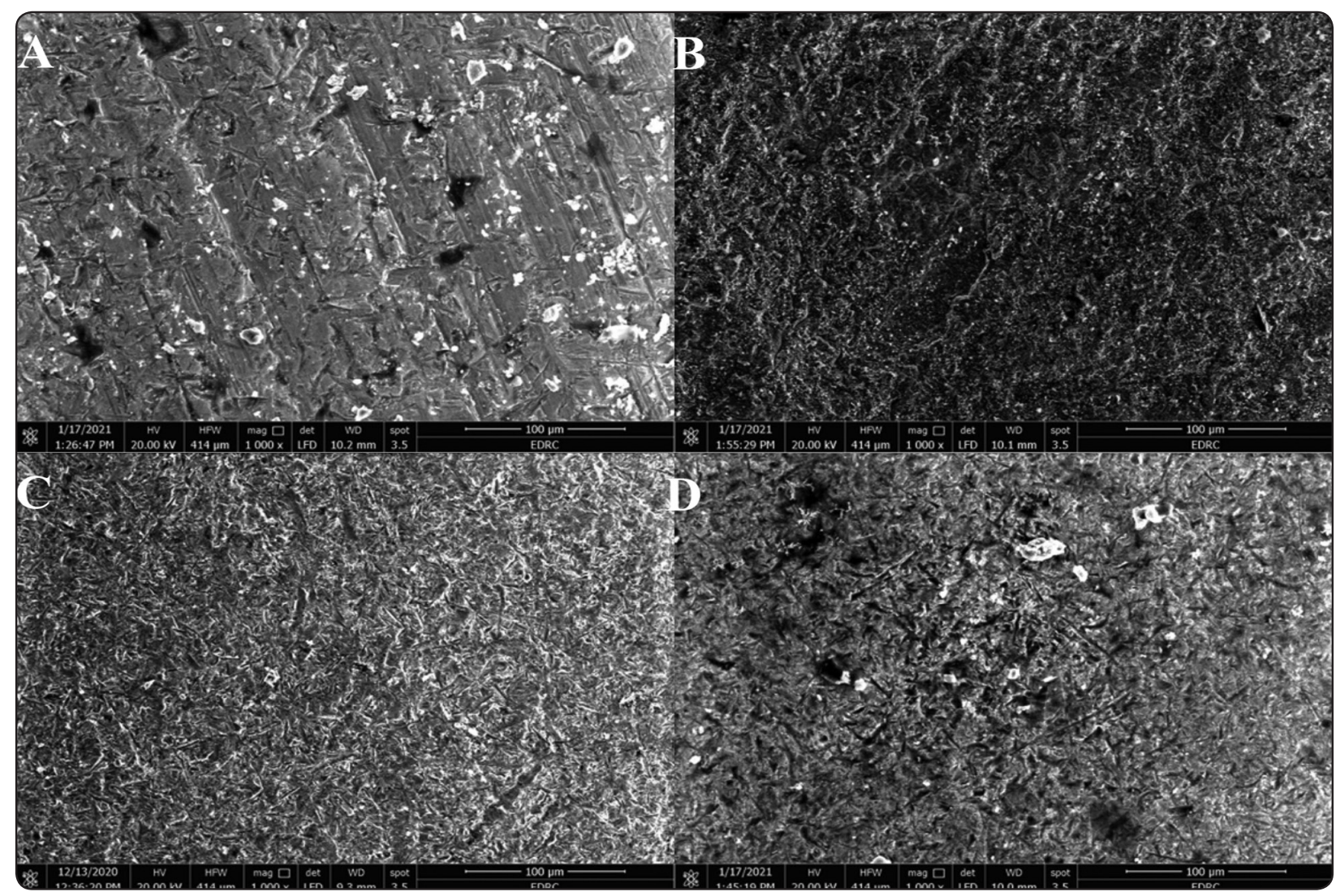

Fig. (4) Scanning electron microscope photos represents surface topography of zirconia specimens with 4 bars air particle abrasion. A: Opaque zirconia; the surface showed multiple striated shallow grooves. B: High translucent zirconia; the surface became very rough with multiple deep grooves and deeper pores. C: Ultra translucent Zirconia; the surface showed relatively roughness with micro-retentive grooves. D: Supra translucent zirconia; the surface showed many rough cracks with multiple relatively deep scars and grooves. 


\section{DISCUSSION}

Translucency, natural tooth color, and outstanding light transmission in addition to optimal mechanical properties, are needed to create highly esthetic all-ceramic restoration. Due to up to date zirconia with excellent mechanical properties, tooth-colored and natural appearance, and low plaque accumulation, it is considered a good choice for dental restorations. (Gracis et al. 2015). It is proven that adhesion between zirconia and adhesive resin cements is a difficult task and reply highly on zirconia structure and surface treatment. (Tzanakakis et al. 2016).

Zirconia could be classified according to yttria content, which increases the grain size into the following main groups; opaque, 3 mole \% Y-TZP (mainly tetragonal), the second group is more translucent, 4 mole \% Y-TZP, the third group is most translucent, 5 mole \% Y-TZP(Chen et al. 2016; Kelly and Denry. 2008). As compared to opaque 3 Y-TZP, the second and third groups have $25 \%$ to $50 \%$ cubic polycrystals, which lower fracture toughness and flexural strength and increase light transmission due to increased cubic phase, less porosity, and larger cubic grains. (Zhang et al. 2019).

This study compared the different types of monolithic zirconia opaque, high, ultra and supra translucent zirconia owing to their structural and compositional difference, and different air particle abrasion pressure to their shear bond strength after applying the zirconia primer, and thus with the increase in the translucency there is an increase in the Yttria \% and a decrease in the Alumina content (Zhang et al. 2015, Kontonasaki et al. 2020).

In this study air abrasion was used as it was proven that it provides micromechanical bonding and that the use of 10-methacryloyloxydecyl-dihydrogenphosphate (MDP)-base primers strengthens the bonds, as it provides a chemical bond with the oxide layer of zirconia (Colombo et al. 2020).

Multiple techniques were used in the current study to protect and standardize the study's goal. Instead of crowns or veneers, square-shaped specimens were prepared. Natural teeth were not used to bond the zirconia square shaped specimens for SBS test. Since each natural tooth has a history regarding to age of patient, degree of calcification, dentinal tubules count, amount of moisture content, date of extraction, etc. All these factors are extremely difficult to control and standardize and thus may affect the integrity of results. Consequently, in current study composite cuboids were used to be bonded to zirconia as an alternative to natural teeth.

Because of the uniform structure of composite resin, which has a remarkably similar modulus of elasticity to dentin and is used on a regular basis as a restoration or build-up of teeth, plexi-frames were created to standardise the size and precisely cement all specimens in exact place. (Panitiwat and Salimee. 2017).

Ultimately, the size of alumina particle chosen in this study was $50 \mu \mathrm{m}$, as sujested by many authors for its ideal contribution surface roughness and monoclinic phase, in addition to producing optimal shear bond strength, and cleansing effect to the fitting surface of the restoration. (Moon et al. 2016, Grasel et al. 2018, Prasad et al. 2017)

The present study aimed to evaluate the effect of increasing APA pressure (from 2 to 4 bars) while using MDP containing primer on 4 different types of zirconia. The study showed that high translucent (3 Y-TZP) zirconia (reduced alumina content from 0.25 weight $\%$ to 0.05 weight $\%$ ) had the highest shear bond strength after using 2 bars $(33.2 \pm 8.4)$ and 4 bars $(37.6 \pm 8.6)$ APA in comparison to all other groups, where it was the same finding in the SEM image of high translucent zirconia with 4 bars APA application where it showed the surface became very rough with multiple deep grooves and deeper pores.

Results showed that application of APA with 4 bars enhance the shear bond strength than 2 bars in all tested groups with significant difference in the opaque $(\mathrm{P}=0.028)$ and supra- translucent 
zirconia $(\mathrm{P}=0.001)$ groups. These finding were contributed with the images of the SEM, where 4 bars APA application images showed more surface irregularities, and deeper pores than 2 bars images.

On the other hand, other studies showed that bond strength for translucent zirconia was equivalent to that of conventional zirconia because both materials generated similar results (Le et al. 2019).

In contrary the more yttria containing zirconia (4 Y-TZP is zirconia containing 4 mole $\%$ and $5 \mathrm{Y}$ TZP zirconia contains 5 mole \%); as the case with the Ultra- translucent and Supra translucenct zirconia specimens, showed lower SBS with the lowest score by the supra translucent in comparison to high translucent zirconia. This could be attributed to the fact that, despite of using a MDP containing adhesive monomer, that should give better bond strength to zirconia than do other systems, yttria markedly improved translucency, but strength was affected because cubic zirconia does not undergo stress-induced transformation (Zhang et al. 2016). The images of the SEM revealed the same results as Ultra translucent and Supra translucent zirconia with 2 or 4 bars APA showed less mechanical irregularities on the surface than high translucent zirconia.

The data obtained support rejection of the null hypothesis, as there was statistically significant difference among the results of the tested groups.

Future attempts should focus on improving translucency of zirconia materials without unduly compromising mechanical integrity.

\section{CONCLUSION}

In the pursuit of aesthetic zirconia, it is well to remain cognizant of reaching better mechanical properties as well. Of particular note is the significant lower values of shear bond strength in successive, more translucent generations, forewarning the necessity for greater caution in clinical implementation.

\section{REFERENCES}

- $\quad$ Ågren, M., Kou, W., \& Molin Thorén, M. (2019). Bond strength of surface-treated novel high translucent zirconia to enamel. Biomaterial Investigations in Dentistry, 6(1), $35-42$.

- Cardoso, J. A., Almeida, P. J., Fischer, A., \& Phaxay, S. L. (2012). Clinical decisions for anterior restorations: the concept of restorative volume. Journal of Esthetic and Restorative Dentistry, 24(6), 367-383.

- Chen, Y. W., Moussi, J., Drury, J. L., \& Wataha, J. C. (2016). Zirconia in biomedical applications. Expert Review of Medical Devices, 13(10), 945-963.

- Colombo, M., Gallo, S., Padovan, S., Chiesa, M., Poggio, C., \& Scribante, A. (2020). Influence of Different Surface Pretreatments on Shear Bond Strength of an Adhesive Resin Cement to Various Zirconia Ceramics. Materials, 13(3), 652.

- $\quad$ El Zayat, S.A., Younis, J.F., \& Wahsh, M.M. (2020). Shear Bond Strength of Translucent Zirconia and Lithium-Disilicate Glass Ceramic to Dentin using Different Surface Treatments. International Journal of Dentistry and Oral Health, 6(2), 29-36.

- Gracis, S., Thompson, V. P., Ferencz, J. L., Silva, N. R., $\&$ Bonfante, E. A. (2015). A new classification system for all-ceramic and ceramic-like restorative materials. International Journal of Prosthodontics, 28(3), 227-235.

- Grasel R, Santos MJ, Chagas Rêgo HM, Rippe MP, Valandro LF; Effect of Resin Luting Systems and Alumina Particle Air Abrasion on Bond Strength to Zirconia. Oper Dent 1 May 2018; 43 (3): 282-290.

- $\quad$ Kelly, J. R., \& Denry, I. (2008). Stabilized zirconia as a structural ceramic: an overview. Dental materials, 24(3), 289-298.

- Kontonasaki, E., Giasimakopoulos, P., \& Rigos, A. E. (2020). Strength and aging resistance of monolithic zirconia: an update to current knowledge. Japanese Dental Science Review, 56(1), 1-23.

- Le, M., Larsson, C., \& Papia, E. (2019). Bond strength between MDP-based cement and translucent zirconia. Dental Materials Journal, 38(3), 480-489.

- Matsuzaki, F., Sekine, H., Honma, S., Takanashi, T., Furuya, K., Yajima, Y., \& Yoshinari, M. (2015). Translucency and flexural strength of monolithic translucent zirconia and porcelain-layered zirconia. Dental Materials Journal, 34(6), 910-917. 
- Moon, J. E., Kim, S. H., Lee, J. B., Han, J. S., Yeo, I. S., \& Ha, S. R. (2016). Effects of airborne-particle abrasion protocol choice on the surface characteristics of monolithic zirconia materials and the shear bond strength of resin cement. Ceramics International, 42(1), 1552-1562.

- Nistor, L., Grădinaru, M., Rîcă, R., Mărășescu, P., Stan, M., Manolea, H., ... \& Moraru, I. (2019). Zirconia use in dentistry-manufacturing and properties. Current Health Sciences Journal, 45(1), 28-35.

- Panitiwat, P., \& Salimee, P. (2017). Effect of different composite core materials on fracture resistance of endodontically treated teeth restored with FRC posts. Journal of Applied Oral Science, 25(2), 203-210.

- Prasad HA, Pasha N, Hilal M, Amarnath GS, Kundapur V, Anand M, Singh S. To Evaluate Effect of Airborne Particle Abrasion using Different Abrasives Particles and Compare Two Commercial Available Zirconia on Flexural Strength on Heat Treatment. Int J Biomed Sci. 2017 Jun;13(2): 93-112.

- Thompson, J. Y., Stoner, B. R., Piascik, J. R., \& Smith, R. (2011). Adhesion/cementation to zirconia and other non-silicate ceramics: where are we now?. Dental Materials, 27(1), 71-82.

- Tzanakakis, E. G. C., Tzoutzas, I. G., \& Koidis, P. T. (2016). Is there a potential for durable adhesion to zirconia restorations? A systematic review. The Journal of Prosthetic Dentistry, 115(1), 9-19.

- Yagawa, S., Komine, F., Fushiki, R., Kubochi, K., Kimura, F., \& Matsumura, H. (2018). Effect of priming agents on shear bond strengths of resin-based luting agents to a translucent zirconia material. Journal of Prosthodontic Research, 62(2), 204-209.

- $\quad$ Yang, B., Barloi, A., \& Kern, M. (2010). Influence of airabrasion on zirconia ceramic bonding using an adhesive composite resin. Dental Materials, 26(1), 44-50.

- $\quad$ Zhang, F., Inokoshi, M., Batuk, M., Hadermann, J., Naert, I., Van Meerbeek, B., \& Vleugels, J. (2016). Strength, toughness and aging stability of highly-translucent Y-TZP ceramics for dental restorations. Dental Materials, 32(12), e327-e337.

- Zhang, F., Reveron, H., Spies, B. C., Van Meerbeek, B., \& Chevalier, J. (2019). Trade-off between fracture resistance and translucency of zirconia and lithium-disilicate glass ceramics for monolithic restorations. Acta Biomaterialia, 91, 24-34.

- Zhang, F., Vanmeensel, K., Inokoshi, M., Batuk, M., Hadermann, J., Van Meerbeek, B., ... \& Vleugels, J. (2015). Critical influence of alumina content on the low temperature degradation of 2-3 $\mathrm{mol} \%$ yttria-stabilized TZP for dental restorations. Journal of the European Ceramic Society, 35(2), 741-750. 